\title{
Tuberculosis Treatment Monitoring by Video Directly Observed Therapy in 5 Health Districts, California, USA
}

\author{
Richard S. Garfein, Lin Liu, Jazmine Cuevas-Mota, Kelly Collins, Fatima Muñoz, \\ Donald G. Catanzaro, Kathleen Moser, Julie Higashi, Teeb Al-Samarrai, Paula Kriner, \\ Julie Vaishampayan, Javier Cepeda, Michelle A. Bulterys, Natasha K. Martin, Phillip Rios, Fredric Raab
}

We assessed video directly observed therapy (VDOT) for monitoring tuberculosis treatment in 5 health districts in California, USA, to compare adherence between 174 patients using VDOT and 159 patients using in-person directly observed therapy (DOT). Multivariable linear regression analyses identified participant-reported sociodemographics, risk behaviors, and treatment experience associated with adherence. Median participant age was 44 (range 18-87) years; $61 \%$ of participants were male. Median fraction of expected doses observed (FEDO) among VDOT participants was higher (93.0\% [interquartile range (IQR) 83.4\%-97.1\%]) than among patients receiving DOT (66.4\% [IQR 55.1\%89.3\%]). Most participants (96\%) would recommend VDOT to others; $90 \%$ preferred VDOT over DOT. Lower FEDO was independently associated with US or Mexico birth, shorter VDOT duration, finding VDOT difficult, frequently taking medications while away from home, and having video-recording problems $(p<0.05)$. VDOT cost $32 \%$ (range $6 \%-46 \%$ ) less than DOT. VDOT was feasible, acceptable, and achieved high adherence at lower cost than DOT.

$\mathrm{T}$ uberculosis (TB) incidence rates in the United States increased slightly in 2015 after 20 years of decline (1). California has the third-highest TB incidence and the

Author affiliations: University of California San Diego, La Jolla, California, USA (R.S. Garfein, L. Liu, J. Cuevas-Mota,

K. Collins, F. Muñoz, J. Cepeda, M.A. Bulterys, N.K. Martin, P. Rios, F. Raab); University of Arkansas, Fayetteville, Arkansas, USA (D.G. Catanzaro); San Diego County Health and Human Services Agency, San Diego, California, USA (K. Moser); San Francisco Department of Public Health, San Francisco, California, USA (J. Higashi); Santa Clara County Public Health Department, San Jose, California, USA (T. Al-Samarrai); Imperial County Public Health Department, EI Centro, California, USA (P. Kriner); San Joaquin Public Health Services, Stockton, California, USA

(J. Vaishampayan)

DOI: https://doi.org/10.3201/eid2410.180459 most TB cases in the United States (2). Although TB is treatable (3), poor medication adherence leads to ongoing transmission, disease progression, and development of drug-resistant strains. Treating drug-resistant TB requires longer regimens with costlier, more toxic, and less effective drugs, highlighting the need for reliable treatment adherence monitoring $(4,5)$. Strict adherence has become increasingly important because new short-course and intermittent treatment regimens have lower tolerance for adherence gaps ( 6 ) and because preventing acquired resistance to new drugs developed to treat multidrug-resistant (MDR) and extensively drug-resistant TB is crucial for preserving gains made in this area (7).

Given the severe consequences of poor adherence, health agencies recommend directly observed therapy (DOT), a process in which healthcare workers or trusted designees watch patients swallow each medication dose $(8-10)$. Although DOT is considered the preferred method for adherence monitoring by health agencies including the World Health Organization (11) and the US Centers for Disease Control and Prevention (12), varying degrees of effectiveness have been reported from delivery of DOT through home visits by DOT workers, patients visiting clinics, and trusted family or community members performing observations (13). Furthermore, the DOT process itself can hinder treatment because of its high cost, personnel requirements, potential for stigma, impact on patient income and mobility, and travel required by patients or healthcare workers (14).

These barriers to DOT prompted some US TB programs to use videoconferencing technology through videophones, computers, or smartphones to remotely observe patients swallowing pills $(15,16)$. This live (synchronous) approach became known as video directly observed therapy (VDOT). Studies of synchronous VDOT indicate that patients adhere to their regimens and mostly prefer VDOT over in-person DOT and that VDOT saves TB programs 
money by reducing travel and personnel costs (17-19). However, barriers such as limiting observation to business hours, network interruptions, and requirements of the Health Insurance Portability and Accountability Act (HIPAA) prompted development of smartphone applications to enable recorded (asynchronous) VDOT. A pilot study in Kenya provided the first published evidence of asynchronous VDOT's acceptance (20). Subsequently, the first study to systematically evaluate asynchronous VDOT among TB patients in San Diego, California, and Tijuana, Mexico, showed that patients and providers found VDOT to be feasible and acceptable, with $>95 \%$ of expected doses observed, but lacked a comparison group (21). We assessed treatment adherence for patients using VDOT versus traditional DOT and evaluated adherence, feasibility, acceptability, and cost differences between urban and rural TB programs.

\section{Methods}

\section{Design}

We conducted a prospective, multisite, single-arm trial in which all participants had TB treatment monitored using asynchronous VDOT. As a comparator, medical record reviews provided adherence data from a sample of patients who were monitored using in-person DOT at the same clinics. All VDOT participants used DOT for the first 2 weeks or until medication tolerance was established (whichever was longer) before initiating VDOT. Participants continued using VDOT until treatment completion or their provider switched them back to DOT.

A University of California-San Diego Institutional Review Board approved this study, as did each participating health department. Study participation did not affect treatment prescribed by participants' physicians.

\section{Population and Recruitment}

The study population consisted of patients receiving DOT for active or suspected pulmonary TB in 3 urban (San Diego, San Francisco, Santa Clara) and 2 rural (San Joaquin, Imperial) California health jurisdictions. Patients $\geq 18$ years of age with no plans to move from the jurisdiction before completing treatment and $\geq 30$ days of treatment remaining were eligible. Patients with MDR TB were eligible; however, only $1.4 \%$ of California's TB patients had MDR TB (22).

TB program staff recruited patients sequentially during routine DOT visits. Research staff explained VDOT and study procedures to interested patients and obtained written informed consent. Asynchronous VDOT was available only to study participants; patients who declined participation continued treatment through DOT. In San Diego and Santa Clara counties, synchronous VDOT was also offered to patients who were unsuitable for DOT. Two patients declined to participate before enrollment, and 5 who initially consented withdrew before starting VDOT.

Historical controls $(n=159)$ were group-matched by age, race or ethnicity, and sex from a random sample of patients at the 5 study sites to obtain estimates of adherence to in-person DOT. To avoid selection bias from using patients who were not offered VDOT, controls were selected from patients who completed TB treatment during the year before asynchronous VDOT introduction at each site.

\section{VDOT Description}

The VDOT application (Figure 1) enabled participants to record themselves swallowing each treatment dose and send videos for review by a DOT worker. Each recorded dose was automatically date- and time-stamped, encrypted, and uploaded to a secure server over a cellular or wireless network. Once the data were received by the server, the smartphone application deleted videos from the device to prevent unintentional disclosure of participant information and conserve device memory. Videos were stored on the smartphone in a manner that prevented viewing, editing, resending, or deleting them to protect participant privacy and ensure video fidelity. The asynchronous design allowed participants to take their medications regardless of network connectivity (e.g., while traveling) because videos uploaded automatically whenever cellular or WiFi connections were established. An application status screen allowed participants to see when videos were uploaded or pending. The system sent daily medication reminders by text message or email. Participants were loaned smartphones with cellular data plans to ensure that the application performed identically for all participants and avoided service outages.

TB program staff trained participants to use VDOT during routine clinic or home visits. As with DOT, whenever possible, participants were seen by staff who spoke their preferred language; otherwise, telephone-based translation services were used. Once participants demonstrated VDOT competency, they were given smartphones and instructed to record their next dose alone at the prescribed time. If the participant or DOT worker had concerns about the procedures, the DOT worker kept the phone and repeated the training during subsequent in-person DOT visits; thus, the number of training days could vary by participant. Participants also received a VDOT reference pamphlet. To minimize health risks, participants were instructed to call or visit their healthcare provider before taking medications when side effects occurred, rather than reporting side effects through videos. DOT workers regularly logged onto a password-protected website to view videos and document their observations. If expected videos were missing or videos did not clearly show participants ingesting medications, participants 


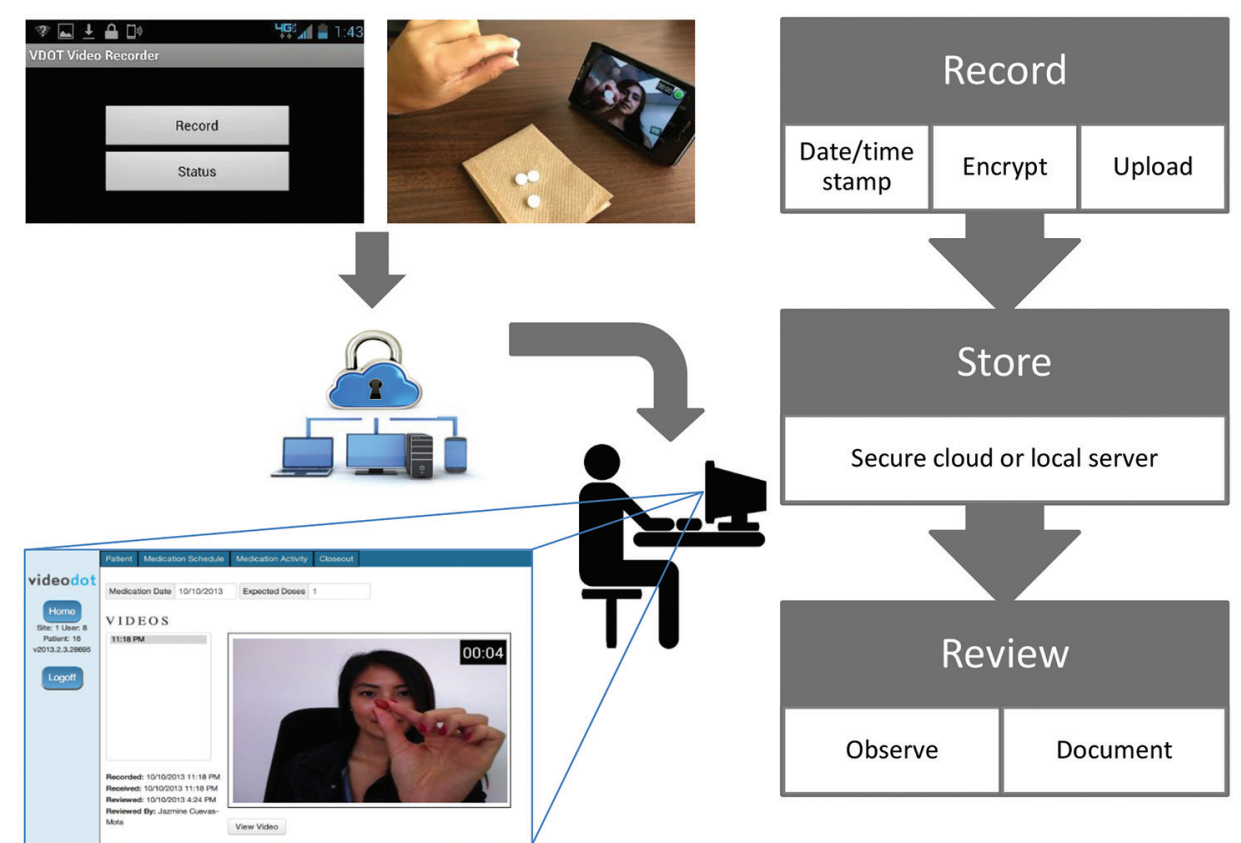

Figure 1. Schematic of asynchronous VDOT in a study assessing VDOT for monitoring tuberculosis treatment, 5 California health districts, 2015-2016. Patients use VDOT smartphone application to record a video of themselves ingesting their medications. After recording stops, the application encrypts the video and transfers it through a cellular or Wi-Fi connection to a server for storage and playback. On a routine basis, treatment monitors log into a secure website to view each video and document their observations. Missing videos or videos not showing complete dose ingestion trigger follow-up procedures to investigate missed doses and provide patient support as needed. VDOT, video directly observed therapy. were contacted to identify problems and provide support as needed. Decisions about returning participants to DOT were made on a case-by-case basis rather than by using strict adherence-based criteria because some missed doses were unavoidable and requiring DOT for participants who could not meet in-person might adversely affect adherence. Routine medication refill and health monitoring visits occurred per standards of care.

\section{Data Collection}

We conducted brief (15-20 minute) baseline (before initiating VDOT) and follow-up (after ending VDOT) telephone interviews to assess sociodemographic variables, experience using mobile technology, TB history and risk factors, privacy concerns, and perceptions of TB treatment monitoring. Research staff, rather than care providers, conducted interviews to minimize response bias. Participants received \$10 USD for each interview; no remuneration was paid for sending videos.

To measure treatment adherence among control patients, TB program staff reviewed their DOT records abstracting treatment start and end dates; DOT start and end dates; treatment outcome; and the number of doses expected, observed, and unobserved (i.e., self-administered, not taken, or treatment suspended). DOT was predominantly community-based and required staff travel; however, San Francisco also offered clinic-based DOT to patients who could conveniently access the clinic. Nonclinical personnel conducted most DOT visits; nurses also provided some DOT based on clinical needs and staffing considerations. Control patients were not interviewed.

\section{VDOT versus DOT}

Because VDOT was introduced after participants had initiated treatment using DOT, we calculated the fraction of expected doses observed (FEDO) while the patient was on VDOT as a measure of adherence. FEDO equals the number of observed doses divided by the sum of observed doses, missed doses, and self-administered doses. For each day that medication doses were expected, DOT workers documented whether they observed all, some, or no pills being ingested. For this analysis, doses were only considered observed if all pills were taken. If no video was received or ingestion of fewer than all pills was observed, the dose was considered missed, as were self-administered doses. Because weekend doses are not ordinarily observed in DOT, they were excluded from this calculation. FEDO was calculated for VDOT and control patients. Adherence (doses observed divided by total doses prescribed) was also computed for control patients because they used DOT throughout their treatment.

\section{Cost Analysis}

Employing a healthcare provider perspective, we used an ingredients-based, bottom-up approach $(23,24)$ to estimate the average per-patient cost of a standard, Centers for Disease Control and Prevention-recommended (3), 6-month treatment regimen for drug-susceptible TB using DOT or VDOT. Because the likelihood of medication side effects differs between the intensive (56 daily doses) and continuation (126 daily doses) phases of treatment, costs were stratified by treatment phase and then summed to calculate the overall average patient treatment cost. Nurses from 4 
sites completed a standardized questionnaire assessing personnel time, personnel salaries, and resources required to administer DOT and VDOT. Staff turnover precluded data collection from the fifth site. Completed questionnaires were discussed jointly by teleconference to ensure that all sites interpreted the questions and responded uniformly. Cost data were collected during March-June 2017 and presented in 2017 USD.

DOT personnel costs included time for patient contact, administrative tasks, and travel. VDOT personnel costs included time for community-based visits before initiating VDOT, patient VDOT training, administrative tasks, video observation, and follow-up when expected videos were not received. Some in-person observations also occurred among patients using VDOT because all patients received DOT for $\geq 2$ weeks before starting VDOT and patients in San Francisco were observed in-person during weekly medication refill visits to the clinic. We converted annual salaries, including fringe benefits, into an hourly rate, assuming a 40-hour workweek. All personnel reported fulltime employment. The total time for each DOT-related task (administrative, patient contact, and travel) needed to treat each patient was multiplied by the hourly rate and then summed for all personnel.

To calculate an overall per-patient travel cost, we multiplied the average number of miles per patient visit and total number of in-person visits by the current federal mileage reimbursement rate ( $\$ 0.54$ per mile). This approach conservatively estimated travel costs because it assumed that DOT workers used personal vehicles rather than costlier county-owned vehicles. Because DOT workers often visited multiple patients in a single outing rather than returning to the health department between each patient visit, we calculated the average number of miles per visit by dividing the average number of miles driven per day for DOT-related activities by the average number of in-person visits on any given day.

Corporate prices paid by the investigators for smartphones $(\$ 100)$ and service plans $(\$ 54 /$ month) during the study were applied to all sites. An estimated VDOT application cost of $\$ 35 /$ month/patient was applied on the basis of products commercially available at the time this article was written. Costs of antibiotics, laboratory tests, chest radiographs, and clinical examinations were excluded because they were assumed to be equivalent for VDOT and DOT.

\section{Statistical Analysis}

We used Kruskal-Wallis and Fisher exact tests to determine differences in sociodemographic characteristics, TB history, TB risk factors, and VDOT perception variables across study sites. We assessed associations between FEDO and independent variables by using Kruskal-Wallis tests (categorical variables), Wilcoxon rank sum tests (binary variables), and Spearman correlation coefficients (continuous variables). We used simple linear regression to identify factors associated with FEDO and considered significant variables $(p<0.15)$ for inclusion in multivariable linear regression analysis. We used backward stepwise elimination to remove nonsignificant variables until only variables with $\mathrm{p}<0.05$ remained in the final model and assessed normal assumption of residuals by using normal probability plot, and influential observations were assessed by residuals and Cook's distance. We performed Wilcoxon rank sum tests to compare FEDO between VDOT and DOT and used R statistical software (25) to conduct analyses.

\section{Results}

\section{Participant Characteristics and VDOT Perceptions}

Overall, 274 participants (248 urban and 26 rural) enrolled during October 2014-October 2015 contributed adherence and baseline interview data (Table 1). Median participant age was 44 (range 18-87) years; $61 \%$ were male, $57 \%$ were Asian, 30\% were Hispanic or Latino, and $7 \%$ were white. Most (67\%) were born in other countries (predominantly countries in Asia), followed by the United States $(17 \%)$ and Mexico (16\%). Education and income were low overall, but most participants had health insurance. Most participants (90\%) owned cell phones; $72 \%$ owned smartphones. Substance use, other than smoking (42\%), was uncommon, and no participants were homeless. Only race or ethnicity, education level, and country of birth differed across sites $(\mathrm{p}<0.05)$.

We obtained VDOT observation data from the 274 enrolled participants, 214 (78\%) of whom completed follow-up interviews (Table 2). Twenty-seven percent of participants reported not sharing their VDOT experience with family members, and $66 \%$ did not share with others. Although 34\% disclosed having concerns about being seen recording VDOT videos, only $8 \%$ failed to record $>1$ dose because of privacy concerns. At follow-up, only $2 \%$ of participants thought VDOT was less confidential than DOT, and $96 \%$ reported that VDOT was "very or somewhat easy to perform"; only 3\% would choose DOT over VDOT if they had to repeat treatment, and $96 \%$ would recommend VDOT to other patients. Training VDOT procedures to participants took a median of 1 day across sites; $74 \%$ of participants required 1 day, whereas $4 \%$ needed $\geq 4$ days (data not shown). Only $12(4.4 \%)$ participants were returned to DOT before completing treatment because of poor adherence $(n=5)$, a lost or broken phone $(n=4)$, or technical or connectivity problems $(n=3)$.

\section{FEDO by Treatment Monitoring Method}

Study participants used VDOT a median of 5.4 months (interquartile range [IQR] 3.5-7.1 months), generating 42,211 
Table 1. Baseline characteristics of patients participating in a study assessing VDOT for monitoring tuberculosis treatment, by site, 5 California health districts, 2015-2016*

\begin{tabular}{|c|c|c|c|c|c|c|c|}
\hline \multirow[b]{2}{*}{ Characteristic } & \multirow[b]{2}{*}{ Total } & \multicolumn{5}{|c|}{ Site } & \multirow[b]{2}{*}{$\mathrm{p}$ value $\dagger$} \\
\hline & & San Diego & $\begin{array}{c}\text { San } \\
\text { Francisco }\end{array}$ & Santa Clara & Imperial & $\begin{array}{c}\text { San } \\
\text { Joaquin }\end{array}$ & \\
\hline No. patients & 272 & 99 & 99 & 49 & 10 & 15 & \\
\hline \multicolumn{8}{|l|}{ Age, y } \\
\hline Mean (SD) & $43.8(16.5)$ & $42.0(16.9)$ & $46.5(15.5)$ & $42.2(16.2)$ & $46.6(22.0)$ & $41.7(16.1)$ & 0.19 \\
\hline Range & $18-87$ & $18-87$ & $24-86$ & $21-83$ & $21-69$ & $21-76$ & \\
\hline \multicolumn{8}{|l|}{ Education } \\
\hline$\leq$ Primary school & $26(10)$ & $10(10)$ & $12(12)$ & $3(6)$ & $1(10)$ & 0 & 0.02 \\
\hline High school & $105(39)$ & $39(40)$ & $39(40)$ & $13(27)$ & $5(50)$ & $9(60)$ & \\
\hline Some college or technical school & $67(25)$ & $25(26)$ & $17(18)$ & $15(31)$ & $4(40)$ & $6(40)$ & \\
\hline$\geq$ Bachelor's degree & $71(26)$ & $24(24)$ & $29(30)$ & $18(37)$ & 0 & 0 & \\
\hline \multicolumn{8}{|l|}{ Sex } \\
\hline M & $167(61)$ & $59(60)$ & $61(62)$ & $34(69)$ & $5(50)$ & $8(53)$ & 0.65 \\
\hline $\mathrm{F}$ & $105(39)$ & $40(40)$ & $38(38)$ & $15(31)$ & $5(50)$ & $7(47)$ & \\
\hline \multicolumn{8}{|l|}{ Race or ethnicity } \\
\hline Asian & $154(57)$ & $41(41)$ & $68(69)$ & $37(76)$ & $10(10)$ & $7(47)$ & $<0.001$ \\
\hline Caucasian or white & $19(7)$ & $7(7)$ & $7(7)$ & $1(2)$ & 0 & $4(27)$ & \\
\hline Hispanic or Latino & $82(30)$ & $42(42)$ & $21(21)$ & $6(12)$ & $9(90)$ & $4(27)$ & \\
\hline Other‡ & $17(6)$ & $9(9)$ & $3(3)$ & $5(10)$ & 0 & 0 & \\
\hline \multicolumn{8}{|l|}{ Country of birth } \\
\hline United States & $47(17)$ & $22(22)$ & $9(9)$ & $4(8)$ & $4(40)$ & $8(53)$ & $<0.001$ \\
\hline Mexico & $44(16)$ & $26(26)$ & $7(7)$ & $5(10)$ & $5(50)$ & $1(7)$ & \\
\hline Other§ & $181(67)$ & $51(52)$ & $83(84)$ & $40(82)$ & $1(10)$ & $6(40)$ & \\
\hline \multicolumn{8}{|l|}{ Annual household income, USD } \\
\hline$<10,000$ & $110(44)$ & $43(46)$ & $43(47)$ & $13(29)$ & $6(55)$ & $8(57)$ & 0.09 \\
\hline $10,000-30,000$ & $74(30)$ & $24(28)$ & $29(32)$ & $15(33)$ & $3(27)$ & $3(21)$ & \\
\hline $30,000-50,000$ & $26(10)$ & $13(15)$ & $9(10)$ & $2(4)$ & $0(0)$ & $2(14)$ & \\
\hline$>50,000$ & $39(16)$ & $10(11)$ & $11(12)$ & $15(33)$ & $2(18)$ & $1(7)$ & \\
\hline Had health insurance, yes vs. no & $229(85)$ & $76(78)$ & $85(86)$ & $43(90)$ & $10(91)$ & $15(100)$ & 0.12 \\
\hline Owned cell phone, yes vs. no & $247(90)$ & $90(91)$ & $92(93)$ & $44(90)$ & $9(82)$ & $12(80)$ & 0.33 \\
\hline Owned smartphone, yes vs. no & $196(72)$ & $71(72)$ & $67(68)$ & $41(84)$ & $7(64)$ & $10(67)$ & 0.26 \\
\hline Homeless, yes vs. noI & 0 & 0 & 0 & 0 & 0 & 0 & NA \\
\hline Ever smoked cigarettes, yes vs. no & $116(42)$ & $43(43)$ & $41(41)$ & $17(35)$ & $7(64)$ & $8(53)$ & 0.41 \\
\hline Marijuana use, yes vs. noI & $18(7)$ & $5(5)$ & $7(7)$ & $2(4)$ & $2(18)$ & $2(13)$ & 0.26 \\
\hline Noninjection drug use, yes vs. noI & $4(1)$ & $1(1)$ & $2(2)$ & $1(2)$ & 0 & 0 & 1 \\
\hline Ever iniection druq use, yes vs, no & $3(1)$ & $1(1)$ & 0 & $1(2)$ & $1(9)$ & 0 & 0.07 \\
\hline \multicolumn{8}{|c|}{$\begin{array}{l}\text { *Values are no. (\%) participants unless otherwise indicated. VDOT, video directly observed therapy; NA, not applicable. } \\
\text { tp values based on Fisher exact test or Kruskal-Wallis test. Variable totals might not sum to column totals because of missing data. } \\
\text { †Other race group includes African American }(n=3) \text {, American Indian }(n=2) \text {, Pacific Islander }(n=1) \text {, and mixed and other races }(n=11) \text {. } \\
\text { §Other countries were predominantly in Asia. } \\
\text { đReferent period is the previous } 6 \text { months. }\end{array}$} \\
\hline
\end{tabular}

videos (Table 2). Median FEDO was 93.0\% (IQR 83.4\%$97.1 \%$ ), compared with $66.4 \%$ (IQR $55.1 \%-89.3 \%$ ) for control patients using only DOT (Figure 2). By contrast, median adherence was 100\% (IQR 97.0\%-100\%) for control patients because of an unwavering commitment by TB program staff to ensure patients completed their treatment.

\section{Correlates of FEDO}

Median FEDO differed across individual sites (range $84.5 \%-96.1 \%$; $p<0.001$ ); however, the extreme values occurred in the 2 rural sites (Table 2). Thus, FEDO did not differ between the combined urban and rural sites $(92.8 \%$ vs. $94.2 \% ; p=0.51$ ) in bivariate analysis (Table 3, https:// wwwnc.cdc.gov/EID/article/24/10/18-0459-T3.htm). FEDO differed by race or ethnicity and country of birth, increased with longer VDOT use and higher annual income, and decreased with marijuana use in the prior 6 months. Participants who found VDOT more difficult, more often took medications while away from home, more often had problems using the VDOT application, and more often had problems uploading videos because of poor network connectivity had lower FEDOs.

In multivariable analysis (Table 4), higher FEDO was independently associated with longer duration of VDOT use. Lower FEDO was associated with birth in Mexico or the United States compared with other countries; feeling VDOT was somewhat or very difficult compared with very easy; taking medication away from home most or every time compared with never; and having problems using VDOT more than half the time compared with "never."

\section{VDOT versus DOT Costs}

The estimated cost for monitoring a 6-month treatment regimen using VDOT (Table 5) varied by site (range \$3,031$\$ 3,911$ ) and was $6 \%-46 \%$ cheaper than community-based 
DOT (range \$3,212-\$5,788) across sites. Reduced personnel costs drove savings, which offset smartphonerelated costs.

\section{Discussion}

VDOT was feasible and acceptable for monitoring TB medication ingestion in urban and rural California health districts. A higher proportion of expected doses was observed as scheduled among VDOT participants than among in-person DOT participants, resulting in shorter treatment duration.

Median FEDO for VDOT was lower than previously reported (95\%) (21), possibly because the earlier study oversampled low-risk patients during the first trial of the VDOT application. Alternatively, the disparity could be attributable to our conservative approach to calculating

Table 2. Reported experiences of patients participating in a study assessing VDOT for monitoring tuberculosis treatment, by site, 5 California health districts, 2015-2016*

\begin{tabular}{|c|c|c|c|c|c|c|c|}
\hline \multirow[b]{2}{*}{ Characteristic } & \multirow[b]{2}{*}{ Total } & \multicolumn{5}{|c|}{ Site } & \multirow[b]{2}{*}{$\mathrm{p}$ value } \\
\hline & & San Diego & $\begin{array}{c}\text { San } \\
\text { Francisco }\end{array}$ & Santa Clara & Imperial & San Joaquin & \\
\hline No. patients & $274 \ddagger$ & 100 & 99 & 49 & 11 & 15 & \\
\hline \multicolumn{8}{|l|}{ VDOT use } \\
\hline Months on VDOT, median (IQR) & $5.4(3.5-7.1)$ & $5.2(3.2-6.3)$ & $5.4(3.5-7.3)$ & $5.5(4.1-8.1)$ & $4.0(2.1-5.6)$ & $6.1(4.4-7.7)$ & 0.08 \\
\hline FEDO, median (SD), IQR & $\begin{array}{c}93.0(13.5) \\
83-97\end{array}$ & $\begin{array}{c}88.7(15.1) \\
77-94\end{array}$ & $\begin{array}{c}95.5(11.8) \\
87-98\end{array}$ & $\begin{array}{c}95.2(10.3) \\
89-98\end{array}$ & $\begin{array}{c}84.5(20.0) \\
78-94\end{array}$ & $\begin{array}{c}96.1(7.9) \\
93-98\end{array}$ & $<0.001$ \\
\hline No. patients in follow-up interviews & 214 & 74 & 84 & 39 & 9 & 7 & \\
\hline \multicolumn{8}{|c|}{ Tuberculosis and treatment perceptions } \\
\hline \multicolumn{8}{|c|}{ Did you share your VDOT experience with family members? } \\
\hline Yes & $156(73)$ & $55(74)$ & $55(66)$ & $30(77)$ & $9(100)$ & $6(86)$ & 0.18 \\
\hline No & $58(27)$ & $19(26)$ & $29(34)$ & $9(23)$ & 0 & $1(14)$ & \\
\hline \multicolumn{8}{|c|}{ Did you share your VDOT experience with friends, neighbors, classmates, or coworkers? } \\
\hline Yes & $73(34)$ & $24(32)$ & $28(33)$ & $13(33)$ & $3(33)$ & $5(71)$ & 0.38 \\
\hline No & $141(66)$ & $50(68)$ & $57(67)$ & $26(67)$ & $6(67)$ & $2(29)$ & \\
\hline \multicolumn{8}{|c|}{ Were you concerned someone would see you using the VDOT cell phone? } \\
\hline Yes & $73(34)$ & $19(26)$ & $34(40)$ & $15(38)$ & $4(44)$ & $1(14)$ & 0.23 \\
\hline No & $141(66)$ & $55(74)$ & $51(60)$ & $24(62)$ & $5(56)$ & $6(86)$ & \\
\hline \multicolumn{8}{|c|}{ Did you ever fail to record a video because you were worried someone was watching you? } \\
\hline Yes & $18(8)$ & $7(9)$ & $9(11)$ & $2(5)$ & 0 & 0 & 0.87 \\
\hline No & $196(92)$ & $67(91)$ & 76 (89) & $37(95)$ & $9(100)$ & $7(100)$ & \\
\hline \multicolumn{8}{|l|}{ Confidentiality of VDOT vs. DOT? } \\
\hline More & $146(70)$ & $49(67)$ & $55(66)$ & $30(77)$ & $7(78)$ & $5(83)$ & 0.68 \\
\hline Less & $5(2)$ & $2(3)$ & $1(1)$ & $2(5)$ & 0 & 0 & \\
\hline Same & $59(28)$ & $22(30)$ & $27(33)$ & $7(18)$ & $2(22)$ & $1(17)$ & \\
\hline \multicolumn{8}{|c|}{ VDOT experience } \\
\hline \multicolumn{8}{|c|}{ Overall, how easy/difficult did you find the VDOT process? } \\
\hline Very easy & $174(81)$ & $58(78)$ & $68(79)$ & $36(92)$ & $6(67)$ & $6(86)$ & 0.19 \\
\hline Somewhat easy & $32(15)$ & $14(19)$ & $13(15)$ & $3(8)$ & $1(11)$ & $1(14)$ & \\
\hline Somewhat or very difficult & $9(4)$ & $2(3)$ & $5(6)$ & 0 & $2(22)$ & 0 & \\
\hline \multicolumn{8}{|c|}{ If you had to redo tuberculosis treatment, would you choose VDOT or DOT? } \\
\hline VDOT & $192(90)$ & $67(92)$ & $75(87)$ & $35(90)$ & $9(100)$ & $6(86)$ & \\
\hline DOT & $6(3)$ & $1(1)$ & $4(5)$ & $1(3)$ & 0 & 0 & 0.9 \\
\hline No preference & $16(7)$ & $5(7)$ & $7(8)$ & $3(8)$ & 0 & $1(14)$ & \\
\hline \multicolumn{8}{|c|}{ Would you recommend VDOT to other tuberculosis patients? } \\
\hline Yes & $202(96)$ & $70(95)$ & $81(96)$ & $35(97)$ & $9(100)$ & $7(100)$ & 0.95 \\
\hline No & $8(4)$ & $4(5)$ & $3(4)$ & $1(3)$ & 0 & 0 & \\
\hline \multicolumn{8}{|c|}{ How often did you take tuberculosis medication away from home? } \\
\hline Never or rarely & $120(56)$ & $39(53)$ & $54(64)$ & $19(49)$ & $5(56)$ & $3(43)$ & 0.36 \\
\hline Less than half or half the time & $48(22)$ & $18(24)$ & $12(14)$ & $13(33)$ & $2(22)$ & $3(43)$ & \\
\hline Most of the time or every time & $46(21)$ & $17(23)$ & $19(22)$ & $7(18)$ & $2(22)$ & $1(14)$ & \\
\hline \multicolumn{8}{|c|}{ How often did you have problems using the VDOT application? } \\
\hline Never & $82(38)$ & $24(32)$ & $41(48)$ & $16(41)$ & $1(11)$ & 0 & 0.06 \\
\hline Rarely & $99(46)$ & $35(47)$ & $33(39)$ & $20(51)$ & $5(56)$ & $6(86)$ & \\
\hline Less than half the time & $23(11)$ & $9(12)$ & $9(11)$ & $2(5)$ & $2(22)$ & $1(14)$ & \\
\hline Half the time or more & $10(5)$ & $6(8)$ & $2(2)$ & $1(3)$ & $1(11)$ & 0 & \\
\hline \multicolumn{8}{|c|}{ How often did poor reception cause you problems uploading videos? } \\
\hline Never & $65(31)$ & $13(18)$ & $34(40)$ & $12(31)$ & $3(33)$ & $3(43)$ & \\
\hline Rarely & $103(49)$ & $41(56)$ & $35(42)$ & $20(51)$ & $3(33)$ & $4(57)$ & 0.15 \\
\hline Less than half the time & $24(11)$ & $11(15)$ & $7(8)$ & $3(8)$ & $3(33)$ & 0 & \\
\hline Half the time or more & $20(9)$ & $8(11)$ & $8(10)$ & $4(10)$ & 0 & 0 & \\
\hline
\end{tabular}




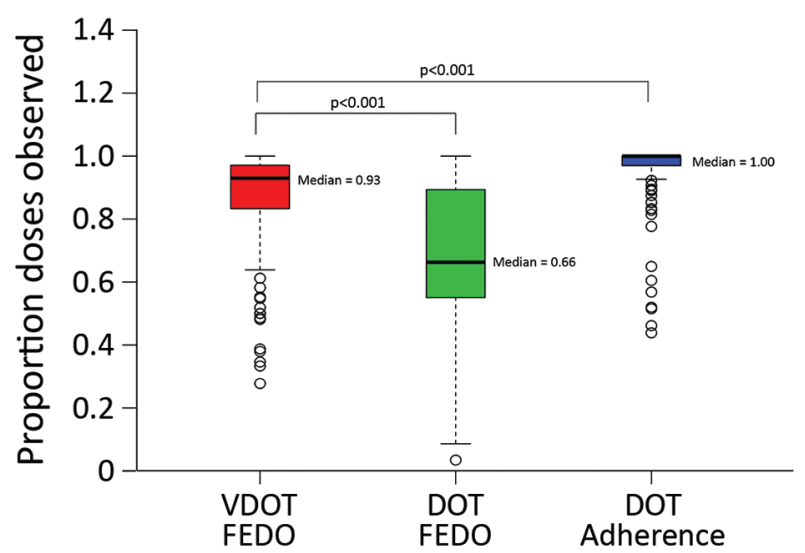

Figure 2. FEDO among patients monitored ingesting medication for tuberculosis by VDOT compared with FEDO and adherence for patients monitored using in-person DOT in a study assessing VDOT for monitoring tuberculosis treatment, 5 California health districts, 2015-2016. FEDO assessed by number of complete doses observed through VDOT divided by the number of doses expected. Adherence assessed by number of doses observed through DOT divided by the number of prescribed doses. Because missed or self-administered doses had to be rescheduled, the number of times a dose was expected could exceed the number of doses prescribed. DOT, directly observed therapy; FEDO, fraction of expected doses observed; VDOT, video directly observed therapy.

FEDO by only counting doses when all pills were taken and treating all doses as missed when a software error caused $5 \%$ of videos received to be unviewable. If unviewable videos and partial doses were counted as observed, FEDO in our study (median 96.4\%, IQR 89\%-99\%) would have matched the prior study.

Defining eligibility criteria for VDOT a priori appears unnecessary. Despite efforts to define VDOT eligibility criteria using these data, only 1 variable known before treatment (country of birth) was associated with FEDO. DOT studies similarly found few predictors of adherence (26). Initial concerns about older patients or those unfamiliar with smartphones having difficulty using VDOT were unfounded because these factors did not predict FEDO. Travel was also not problematic; study staff often reported that patients who traveled or had nontraditional work hours could adhere better after switching to VDOT. Monitoring antiTB therapy involves ongoing communication, negotiation, and cooperation between patients and healthcare providers $(27,28)$, and patient-centered care increased when patients had VDOT as an option. Other than ensuring that patients could tolerate their medications, operate the VDOT application, and access smartphones and service, no evidence was found to support requiring other eligibility criteria.

We observed an association between VDOT problems and lower FEDO, which was driven by only 10 participants who reported having problems half the time or more. Similarly, the association between FEDO and difficulty using VDOT resulted from 9 participants reporting that VDOT was somewhat or very difficult. However, most of these participants encountered the software error described previously, which lowered their FEDO and could explain why they felt VDOT was difficult. Lower FEDO among participants taking medications away from home most or every time could be attributable to difficulty finding a private location to make videos while away from home, which might have also made DOT difficult.

Unlike DOT or synchronous VDOT, asynchronous VDOT enabled patients to take medications outside normal business hours (e.g., at mealtimes or bedtime), which could minimize side effects and improve adherence (16). VDOT also allowed participants to fast during religious holidays, because medication doses could be observed at night after fasting ended. Avoiding intermittent dosing by allowing observations after hours and on weekends and holidays through VDOT could also improve treatment efficacy (29).

Table 4. Multivariable linear regression analysis of factors associated with FEDO among patients treated for tuberculosis, 5 California health districts, 2015-2016*

\begin{tabular}{|c|c|c|c|}
\hline Characteristic & $\begin{array}{c}\text { Beta } \\
\text { coefficient }\end{array}$ & SE & $\mathrm{p}$ value \\
\hline Months on VDOT (per month) & 0.008 & 0.003 & 0.01 \\
\hline \multicolumn{4}{|l|}{ Country of birth (referent: other) } \\
\hline Mexico & -0.095 & 0.022 & $<0.001$ \\
\hline United States & -0.048 & 0.022 & 0.03 \\
\hline \multicolumn{4}{|c|}{ Perceived ease or difficulty of VDOT (referent: very easy) } \\
\hline Somewhat easy & -0.003 & 0.024 & 0.90 \\
\hline Somewhat or very difficult & -0.130 & 0.042 & 0.002 \\
\hline \multicolumn{4}{|c|}{ Took medications while away from home (referent: never or rarely) } \\
\hline Less than half or half the time & -0.004 & 0.020 & 0.83 \\
\hline Most of the time or always & -0.049 & 0.021 & 0.02 \\
\hline \multicolumn{4}{|c|}{ Had problems using the VDOT application (referent: never) } \\
\hline Rarely & -0.001 & 0.018 & 0.97 \\
\hline Less than half the time & -0.040 & 0.029 & 0.16 \\
\hline More than half the time & -0.220 & 0.041 & $<0.001$ \\
\hline
\end{tabular}

${ }^{*} \mathrm{FEDO}$, fraction of expected doses observed $=$ number of complete doses observed through VDOT divided by the number of doses expected; VDOT, video directly observed therapy. 
Tuberculosis Treatment Monitoring, California

Table 5. Average in-person DOT and VDOT costs per treatment course, by site, based on standard drug-susceptible tuberculosis treatment regimen consisting of 56 intensive-phase and 126 continuation-phase doses, 4 California health districts, 2015-2016*

\begin{tabular}{|c|c|c|c|c|}
\hline Characteristic & San Diego & San Francisco & San Joaquin & Imperial \\
\hline \multicolumn{5}{|l|}{ In-person DOT costs } \\
\hline \multicolumn{5}{|l|}{ Personnel } \\
\hline Administrative tasks & 1,038 & 3,043 & 1,913 & 842 \\
\hline In-person patient contact & 1,207 & 622 & 2,223 & 656 \\
\hline Travel & 1,939 & 1,065 & 702 & 1,293 \\
\hline Total personnel (\% of total) & $4,185(91)$ & $4,729(97)$ & $4,838(84)$ & $2,791(87)$ \\
\hline Mileage ( $\%$ of total) & $364(9)$ & $158(3)$ & $950(16)$ & $421(13)$ \\
\hline Grand total & 4,549 & 4,888 & 5,788 & 3,212 \\
\hline \multicolumn{5}{|l|}{ VDOT costs } \\
\hline \multicolumn{5}{|l|}{ Personnel } \\
\hline Administrative tasks & 796 & 1,922 & 1,771 & 1,183 \\
\hline In-person patient contact & 671 & 291 & 393 & 346 \\
\hline Watching videos & 80 & 131 & 152 & 473 \\
\hline Other, e.g., training and follow-up & 869 & 933 & 156 & 348 \\
\hline Total personnel (\% of total) & $2,526(79)$ & $3,277(88)$ & $2,472(83)$ & $2,350(78)$ \\
\hline Mileage (\% of total) & $20(1)$ & 0 & $31(1)$ & $46(2)$ \\
\hline Smartphone costs, device and service (\% of total) & $424(13)$ & $424(7)$ & $424(8)$ & $424(14)$ \\
\hline $\begin{array}{l}\text { VDOT application service fee, } \$ 35 / \mathrm{mo} / \text { patient ( } \% \text { of } \\
\text { total) }\end{array}$ & $210(7)$ & $210(5)$ & $210(7)$ & $210(7)$ \\
\hline Grand total & 3,179 & 3,911 & 3,137 & 3,031 \\
\hline \multicolumn{5}{|l|}{$\%$ Change for VDOT versus in-person DOT } \\
\hline Personnel costs, $\%$ & -40 & -31 & -49 & -16 \\
\hline Overall costs, \% & -30 & -20 & -46 & -6 \\
\hline
\end{tabular}

All sites, except 1, included participants with MDR TB (VDOT duration range 30-537 days) whose adherence was comparable to the cohort overall. Because MDR tuberculosis patients at times require dosing more than once daily, VDOT reduced stress on the TB programs and facilitated quicker return to daily activities for patients on these much longer regimens. Additionally, asynchronous VDOT does not require consistent network connectivity, making it useful for patients in remote areas.

Although asynchronous VDOT offers greater flexibility and reduces self-administered doses, DOT and synchronous VDOT might allow more frequent patient-provider interaction and facilitate patient support. However, asynchronous VDOT could improve case management efficiency by shifting the focus of in-person visits from treatment monitoring, perceived by patients as punitive $(30,31)$, to patient care, support, and other key TB program activities such as contact tracing. The appropriate mix of remote monitoring and direct interaction to support patients throughout treatment remains to be determined with further research. Cost-effectiveness studies are also needed to inform policies around treatment monitoring.

TB risk factors were self-reported and could be underestimated if participants chose not to disclose stigmatized behaviors. Because no patients were homeless, we could not examine this risk factor. Three sites (Santa Clara, Imperial and San Joaquin) had never used asynchronous VDOT previously, potentially promoting conservative patient selection; however, their results were similar to sites with VDOT experience. In addition, San Francisco differed from the other sites by requiring weekly, rather than monthly, refill visits, which could have increased adherence; however, adherence was comparable across sites. Because providers could switch participants from VDOT back to DOT, observed FEDOs could have been skewed upward if nonadherent participants were removed from VDOT early. However, only $12(4.3 \%)$ participants returned to DOT before completing treatment, of whom only 5 did so because of poor adherence. Removing these participants had little effect on FEDO overall. This study was conducted in a highincome country and might not reflect VDOT performance in low- and middle-income countries.

To our knowledge, our study is the largest prospective study of asynchronous VDOT to date. Patients with TB treatment monitored by VDOT had more expected medication doses observed than patients monitored using DOT. VDOT performed similarly in urban and rural health departments, with high observation rates and positive patient perceptions across sites. Although some participants returned to DOT, most were effectively monitored to completion by using VDOT. VDOT reduced TB-control program costs compared with DOT. Other than country of birth, patient characteristics did not predict adherence, suggesting that TB-control programs could offer VDOT broadly and provide additional support, or switch to DOT if adherence declines rather than restricting VDOT use to patients with prespecified characteristics. Asynchronous VDOT was found to be a cost-effective method of monitoring TB treatment in the United States; however, similar studies are needed in countries with high burdens of TB 
and limited resources, where smartphone penetration and cultural acceptance of transmitting personal images over the Internet could differ.

\section{Acknowledgments}

We thank the participants for their contributions to the study. We are also grateful for the cooperation and invaluable feedback provided by each of the participating health departments. The Practice to Policy Project (P3) study group included Rocio Agraz-Lara, Teresa Ampie, Anne Cass, Mario Gutierrez (deceased), Pamela Kennedy, Mei Kwong, Stephanie Le, Krystal Liang, Laura Nasseri, Floreida Quiaoit, Lois Ritter, Laura Romo, Jaspreet Sidhu, Stephanie Spencer, Janice Westenhouse, Jan Young, and Miguel Zamora.

This study was funded by a grant from the California HealthCare Foundation. J.C. was funded by National Institutes of Health grant no. K01-DA043421. N.K.M. was funded by National Institutes of Health grant no. R01-DA037773 and the University of California-San Diego (USCD) Center for AIDS Research grant no. P30-AI036214.

R.S.G. is a cofounder of SureAdhere Mobile Technology, Inc., a VDOT service provider. No funding, software, or other resources were provided by SureAdhere for the study. To mitigate potential conflicts of interest, all interpretation and reporting of the study findings were approved by coauthors who are unaffiliated with SureAdhere. The terms of this arrangement have been reviewed and approved by USCD in accordance with its conflict of interest policies. K.C. began consulting for SureAdhere after the data collection was complete and she was no longer affiliated with USCD.

Author contributions: R.S.G. conceptualized and designed the study, supervised its implementation, drafted the manuscript, and led the writing process. L.L. analyzed the data, interpreted results, and wrote sections of the paper. J.C.-M., K.C., F.M., and M. B. assisted in research staff training, study implementation, data collection, data quality assurance, and manuscript preparation. D.G.C. assisted with management, processing, and analysis of data and contributed to the manuscript. J.C. and N.K.M. conducted the economic analysis and wrote sections of the manuscript. K.M., J.H., T.A-S., P.K., and J.V. supervised study implementation at their respective health departments, ensured fidelity to study protocols, contributed to interpretation of results, and assisted in manuscript preparation and final review. F.R. and P.R. developed, programmed, and maintained the VDOT software application. All authors critically reviewed and approved the manuscript.

\section{About the Author}

Dr. Garfein is an infectious disease epidemiologist at the USCD School of Medicine. His research focuses on the epidemiology and prevention of tuberculosis, HIV, and viral hepatitis among vulnerable populations. His research interests also include developing and evaluating interventions that support efforts to eliminate tuberculosis and other treatable diseases.

\section{References}

1. Salinas JL, Mindra G, Haddad MB, Pratt R, Price SF, Langer AJ Leveling of tuberculosis incidence-United States, 2013-2015. MMWR Morb Mortal Wkly Rep. 2016;65:273-8. http://dx.doi.org/ 10.15585/mmwr.mm6511a2

2. Schmit KM, Wansaula Z, Pratt R, Price SF, Langer AJ. Tuberculosis - United States, 2016. MMWR Morb Mortal Wkly Rep. 2017;66:289-94. http://dx.doi.org/10.15585/ mmwr.mm6611a2

3. Nahid P, Dorman SE, Alipanah N, Barry PM, Brozek JL, Cattamanchi A, et al. Official American Thoracic Society/Centers for Disease Control and Prevention/Infectious Diseases Society of America Clinical Practice Guidelines: Treatment of DrugSusceptible Tuberculosis. Clin Infect Dis. 2016;63:e147-95. http://dx.doi.org/10.1093/cid/ciw376

4. Gandhi NR, Nunn P, Dheda K, Schaaf HS, Zignol M, van Soolingen $\mathrm{D}$, et al. Multidrug-resistant and extensively drug-resistant tuberculosis: a threat to global control of tuberculosis. Lancet. 2010;375:1830-43. http://dx.doi.org/10.1016/ S0140-6736(10)60410-2

5. Falzon D, Gandhi N, Migliori GB, Sotgiu G, Cox HS, Holtz TH, et al.; Collaborative Group for Meta-Analysis of Individual Patient Data in MDR-TB. Resistance to fluoroquinolones and second-line injectable drugs: impact on multidrug-resistant TB outcomes. Eur Respir J. 2013;42:156-68. http://dx.doi.org/ 10.1183/09031936.00134712

6. Mitnick CD, McGee B, Peloquin CA. Tuberculosis pharmacotherapy: strategies to optimize patient care. Expert Opin Pharmacother. 2009;10:381-401. http://dx.doi.org/10.1517/14656560802694564

7. Conradie F, Diacon A, Everitt D, Mendel C, Van Niekerk C, Howell P, et al. The NIX-TB trial of pretomanid, bedaquiline and linezolid to treat XDR-TB. Conference on Retroviruses and Opportunistic Infections; Seattle, Washington, USA; February 13-16, 2017 [cited 2018 Jul 27]. http://www.croiconference.org/sessions/ nix-tb-trial-pretomanid-bedaquiline-and-linezolid-treat-xdr-tb

8. Frieden TR, Sbarbaro JA. Promoting adherence to treatment for tuberculosis: the importance of direct observation. World Hosp Health Serv. 2007;43:30-3.

9. Migliori GB, Zellweger JP, Abubakar I, Ibraim E, Caminero JA, De Vries G, et al. European union standards for tuberculosis care. Eur Respir J. 2012;39:807-19. http://dx.doi.org/10.1183/ 09031936.00203811

10. World Health Organization. Global tuberculosis control: a short update to the 2009 report [cited $2018 \mathrm{Jul}$ 27]. http://www.who.int/ iris/handle/10665/44241

11. World Health Organization. Multidrug and extensively drugresistant TB (M/XDR-TB): 2010 global report on surveillance and response [cited $2018 \mathrm{Jul}$ 27]. http://www.who.int/tb/ features_archive/m_xdrtb_facts/en

12. Centers for Disease Control and Prevention. Treatment for TB disease [cited $2018 \mathrm{Jul}$ 27]. https://www.cdc.gov/tb/topic/treatment/ tbdisease.htm

13. Volmink J, Garner P. Directly observed therapy for treating tuberculosis. Cochrane Database Syst Rev. 2007;4:CD003343.

14. Pope DS, Chaisson RE. TB treatment: as simple as DOT? Int J Tuberc Lung Dis. 2003; 7:611-5.

15. DeMaio J, Schwartz L, Cooley P, Tice A. The application of telemedicine technology to a directly observed therapy program for tuberculosis: a pilot project. Clin Infect Dis. 2001;33:2082-4. http://dx.doi.org/10.1086/324506 
16. Wade VA, Karnon J, Eliott JA, Hiller JE. Home videophones improve direct observation in tuberculosis treatment: a mixed methods evaluation. PLoS One. 2012;7:e50155. http://dx.doi.org/ 10.1371/journal.pone.0050155

17. Mirsaeidi M, Farshidpour M, Banks-Tripp D, Hashmi S, Kujoth C, Schraufnagel D. Video directly observed therapy for treatment of tuberculosis is patient-oriented and cost-effective. Eur Respir J. 2015;46:871-4. http://dx.doi.org/10.1183/09031936.00011015

18. Center for Connected Health Policy. Using telehealth for directly observed therapy in treating tuberculosis [cited $2018 \mathrm{Jul} 27$ ]. http://www.cchpca.org/using-telehealth-directly-observed-therapytreating-tuberculosis

19. Chuck C, Robinson E, Macaraig M, Alexander M, Burzynski J. Enhancing management of tuberculosis treatment with video directly observed therapy in New York City. Int J Tuberc Lung Dis. 2016;20:588-93. http://dx.doi.org/10.5588/ijtld.15.0738

20. Hoffman JA, Cunningham JR, Suleh AJ, Sundsmo A, Dekker D, Vago F, et al. Mobile direct observation treatment for tuberculosis patients: a technical feasibility pilot using mobile phones in Nairobi, Kenya. Am J Prev Med. 2010;39:78-80. http://dx.doi.org/10.1016/j.amepre.2010.02.018

21. Garfein RS, Collins K, Muñoz F, Moser K, Cerecer-Callu P, Raab F, et al. Feasibility of tuberculosis treatment monitoring by video directly observed therapy: a binational pilot study. Int J Tuberc Lung Dis. 2015;19:1057-64. http://dx.doi.org/10.5588/ ijtld.14.0923

22. California Department of Public Health. Report on tuberculosis in California, 2016 [cited 2018 Jul 27]. https://www.cdph.ca.gov/ Programs/CID/DCDC/CDPH\%20Document\%20Library/ TBCB_Report_2016.pdf

23. Drummond MF, Sculpher MJ, Claxton K, Stoddart GL, Torrance GW. Methods for the economic evaluation of health care programmes. 4th ed. Oxford: Oxford University Press; 2015.

24. Chapko MK, Liu CF, Perkins M, Li YF, Fortney JC, Maciejewski ML. Equivalence of two healthcare costing methods: bottom-up and top-down. Health Econ. 2009;18:1188-201. http://dx.doi.org/10.1002/hec.1422

25. R Core Team. R: A language and environment for statistical computing [cited $2018 \mathrm{Jul}$ 27]. https://www.R-project.org

26. Hirsch-Moverman Y, Daftary A, Franks J, Colson PW. Adherence to treatment for latent tuberculosis infection: systematic review of studies in the US and Canada. Int J Tuberc Lung Dis. 2008;12:1235-54.

27. Zhang H, Ehiri J, Yang H, Tang S, Li Y. Impact of communitybased DOT on tuberculosis treatment outcomes: a systematic review and meta-analysis. PLoS One. 2016;11:e0147744 http://dx.doi.org/10.1371/journal.pone.0147744

28. Karumbi J, Garner P. Directly observed therapy for treating tuberculosis. Cochrane Database Syst Rev. 2015;5:CD003343.

29. Kasozi S, Clark J, Doi SA. Intermittent versus daily pulmonary tuberculosis treatment regimens: a meta-analysis. Clin Med Res. 2015;13:117-38. http://dx.doi.org/10.3121/cmr.2015.1272

30. Queiroz EM, De-La-Torre-Ugarte-Guanilo MC, Ferreira KR, Bertolozzi MR. Tuberculosis: limitations and strengths of directly observed treatment short-course. Rev Lat Am Enfermagem. 2012;20:369-77. http://dx.doi.org/10.1590/ S0104-11692012000200021

31. Sagbakken M, Frich JC, Bjune GA, Porter JD. Ethical aspects of directly observed treatment for tuberculosis: a cross-cultural comparison. BMC Med Ethics. 2013;14:25. http://dx.doi.org/ $10.1186 / 1472-6939-14-25$

Address for correspondence: Richard S. Garfein, University of California, San Diego, 9500 Gilman Dr, MC-0725, La Jolla, CA 92093-0507, USA; email: rgarfein@ucsd.edu

\section{March 2017: Tuberculosis and Mycobacteria}

- Epidemiology of Mycobacterium bovis Disease in Humans in England, Wales, and Northern Ireland, 2002-2014

- Three Cases of Neurologic Syndrome Caused by Donor-Derived Microsporidiosis

- Epidemiology of Invasive Haemophilus influenzae Disease, Europe, 2007-2014

- Zika Virus RNA Replication and Persistence in Brain and Placental Tissue

- Spatiotemporal Fluctuations and Triggers of Ebola Virus Spillover

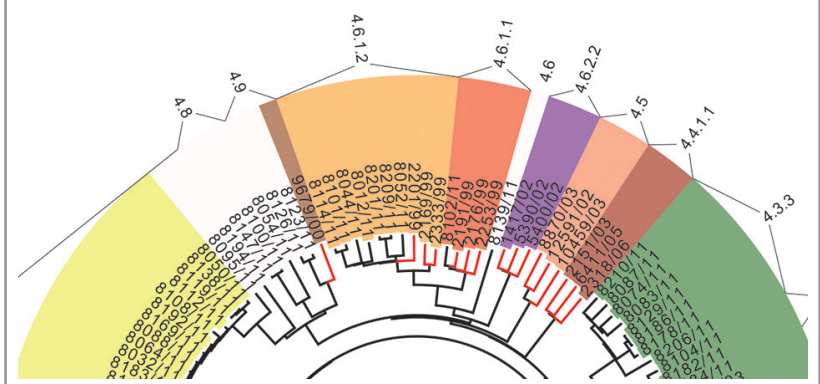

- New Mycobacterium tuberculosis Complex Sublineage, Brazzaville, Congo

- Whole-Genome Analysis of Bartonella ancashensis, a Novel Pathogen Causing Verruga Peruana, Rural Ancash Region, Peru

- Epidemiology of Nontuberculous Mycobacterial Lung Disease and Tuberculosis, Hawaii, USA

- Mycobacterium tuberculosis Transmission among Elderly Persons, Yamagata Prefecture, Japan, 2009-2015

- Comparison of Sputum-Culture Conversion for Mycobacterium bovis and M. tuberculosis

- Use of Mass-Participation Outdoor Events to Assess Human Exposure to Tickborne Pathogens

- Pulmonary Nontuberculous Mycobacteria-Associated Deaths, Ontario, Canada, 2001-2013

https://wwwnc.colc.gov/eid/articles/ issue/23/3/table-of-contents EMERGING WFFECIIOUS DISEASES 